

\title{
A NEW CLASS OF SECANT-LIKE METHODS FOR SOLVING NONLINEAR SYSTEMS OF EQUATIONS
}

\author{
José A. Ezquerro, Angela Grau, Miquel Grau-SÁnchez \\ And Miguel A. HeRnándeZ-Verón
}

\begin{abstract}
Applying twice an idea of Hernández and Rubio (2002) for constructing a oneparameter family of secant-like methods, we define a two-parameter family of secant-like methods for solving nonlinear systems of equations. We analyze the efficiency of this new family and conclude that the Kurchatov method, which is one member of the family, is the most efficient. We illustrate this with Troesch's problem.
\end{abstract}

\section{Introduction}

Iterative methods are typically used for approximating a simple root $\alpha$ of a nonlinear system of equations, say $F(x)=0$, where $F \equiv\left(F_{1}, F_{2}, \ldots, F_{m}\right)$ - each component $F_{i}: D \subseteq \mathbb{R}^{m} \rightarrow \mathbb{R}, i=1,2, \ldots, m$, being defined on a nonempty open convex domain $D$ of $\mathbb{R}^{m}$. The choice of a method for solving $F(x)=0$ usually depends on its efficiency, which links the order of convergence of the method to its computational cost. Two classic measurements of the efficiency, in the sense defined by Traub [25] and Ostrowski [20], are the efficiency index (EI) and the computational efficiency (CE), by

$$
\mathrm{EI}=\rho^{1 / a} \text { and } \mathrm{CE}=\rho^{1 / p},
$$

where $\rho$ is the $R$-order of convergence of the method [21], $a$ represents the number of function evaluations necessary to apply the method and $p$ is the number of multiplications and divisions needed to compute each iteration of the method.

For one-point iterative methods without memory, it is known that the order of convergence $\rho$ is a natural number and can be achieved for methods that depend explicitly on the first $\rho-1$ derivatives of $F$. However, the computational cost increases when it is necessary to calculate successive derivatives.

This work was supported in part by project MTM2011-28636-C02-01 of the Spanish Ministry of Science and Innovation.

MSC2010: 47H99, 65H10.

Keywords: nonlinear equations, iterative methods, divided difference, secant method, Kurchatov method, secant-like method, order of convergence, efficiency index, computational efficiency. 
In this paper, we are interested in numerical methods that avoid the expensive computation of derivatives of $F$ at each step. Among such methods, a popular one is the secant method [2;3], whose algorithm we recall. Given two points $u=\left(u_{1}, \ldots, u_{m}\right)$ and $v=\left(v_{1}, \ldots, v_{m}\right)$ in $\mathbb{R}^{m}$, with $u_{i} \neq v_{i}$ for each $i$, define the (first-order) divided difference of $F$ with respect to $u$ and $v$ as the linear map $[u, v ; F]: \mathbb{R}^{m} \rightarrow \mathbb{R}^{m}$ given by the matrix with the following entries:

$$
\begin{aligned}
{[u, v ; F]_{i j}=\frac{1}{u_{j}-v_{j}} } & \left(F_{i}\left(u_{1}, \ldots, u_{j-1}, u_{j}, v_{j+1}, \ldots, v_{m}\right)\right. \\
& \left.-F_{i}\left(u_{1}, \ldots, u_{j-1}, v_{j}, v_{j+1}, \ldots, v_{m}\right)\right), \quad i, j=1,2, \ldots, m .
\end{aligned}
$$

The secant method prescribes

$$
\left\{\begin{array}{l}
x_{0}, x_{-1} \text { given in } D, \\
x_{n+1}=x_{n}-\left[x_{n-1}, x_{n} ; F\right]^{-1} F\left(x_{n}\right), \quad n \geq 0 .
\end{array}\right.
$$

It is superlinearly convergent with $R$-order of convergence $\frac{1}{2}(1+\sqrt{5})$ [22].

In [13] the authors propose a one-parameter family of secant-like methods for solving $F(x)=0$, containing the secant method and Newton's method. For a given value of the parameter $\lambda \in[0,1]$, the method prescribes

$$
\begin{cases}x_{0}, x_{-1} \text { given in } D, & n \geq 0, \\ y_{n}=\lambda x_{n}+(1-\lambda) x_{n-1}, & n \geq 0 . \\ x_{n+1}=x_{n}-\left[y_{n}, x_{n} ; F\right]^{-1} F\left(x_{n}\right), & \end{cases}
$$

Clearly (3) reduces to the secant method if $\lambda=0$; and, if $F$ is differentiable, (3) reduces to Newton's method for $\lambda=1$, since in this case $[u, v ; F]$ tends to $F^{\prime}(v)$ as $u \rightarrow v$. We know from $[14 ; 15]$ that the $R$-order of convergence of (3) is at least the same as that of the secant method for all $\lambda$. In practice, the closer $x_{n}$ and $y_{n}$, the higher the speed of convergence; indeed, it is shown in [13] that the speed of convergence of (3) increases with $\lambda \in[0,1]$, approaching that of Newton's method when $\lambda$ is close to 1 .

Following the above idea twice, we can generalize the method to two parameters, one for each component of the divided difference involved in the secant method. Given $\gamma, \delta \in \mathbb{R}$, the generalized method prescribes

$$
\begin{cases}x_{0}, x_{-1} \text { given in } D, & n \geq 0 \\ y_{n}=\gamma x_{n}+(1-\gamma) x_{n-1}, & n \geq 0 \\ z_{n}=\delta x_{n}+(1-\delta) x_{n-1}, & n \geq 0 . \\ x_{n+1}=x_{n}-\left[y_{n}, z_{n} ; F\right]^{-1} F\left(x_{n}\right), & \end{cases}
$$

As before we have as particular cases the secant method $(\gamma=0, \delta=1)$ and Newton's method if $F$ is differentiable ( $\gamma=1, \delta=1$ ). The family (4) also contains Kurchatov's method $[4 ; 5 ; 16 ; 24]$, which corresponds to the case $\gamma=0, \delta=2$; explicitly, this 
method prescribes

$$
\left\{\begin{array}{l}
x_{0}, x_{-1} \text { given in } D \\
x_{n+1}=x_{n}-\left[x_{n-1}, 2 x_{n}-x_{n-1} ; F\right]^{-1} F\left(x_{n}\right), \quad n \geq 0 .
\end{array}\right.
$$

In the one-dimensional case, the Kurchatov method has a geometrical interpretation similar to the secant method [4].

The paper is organized as follows. In Section 2, we determine the order of convergence of (4) in terms of $\gamma$ and $\delta$. In Section 3, we compute the efficiencies (EI and CE) and find the parameter values that maximize it. In Section 4.1 we repeat the analysis using a more general efficiency index, CEI, which takes into account both the number of function evaluations and the number of operations. Finally, in Section 4.2, we give an application to Troesch's problem [26], illustrating the theoretical results presented in earlier sections.

To summarize, this paper presents a two-parameter family of iterative methods for solving nonlinear systems of equations that generalizes both the secant method and the Kurchatov method, and shows that, within this family, the Kurchatov method (or in some restricted cases the secant method) is the most efficient.

\section{Order of convergence}

From now on we assume that $F$ is continuously differentiable four times at $\alpha \in D$.

In this section we state and prove Theorem 1, which gives the order of convergence of the family of iterations defined in (4). We start by writing down the development to fourth order of the divided difference of $F$; this was introduced in [8], following ideas from $[11 ; 12]$. See [8] for details.

Thanks to our assumption on the differentiablity of $F$, we can approximate the divided difference by the derivative of $F$, plus corrections up to the fourth derivative:

$$
[y, x ; F]=F^{\prime}(\alpha)+\sum_{k=1}^{3}\left(\frac{1}{(k+1) !} F^{(k+1)}(\alpha) \sum_{i=0}^{k} e^{k-i} \tilde{e}^{i}\right)+W(x, e, \tilde{e}),
$$

where $e=x-\alpha, \tilde{e}=y-\alpha$, the $(k+1)$-st derivative $F^{(k+1)}(\alpha)$ is understood as the appropriate $(k+1)$-linear map acting on the $k$ vectors whose "product" is written under the inner sum, together with the vector on which $[y, x ; F]$ acts, and finally $W(x, e, \tilde{e})$ is a linear map $\mathbb{R}^{m} \rightarrow \mathbb{R}^{m}$ satisfying $\left\|\left[F^{\prime}(\alpha)\right]^{-1} W(x, e, \tilde{e})\right\|=$ $o\left(\|e\|^{p}\|\tilde{e}\|^{q}\right)$, for all $p, q=0,1,2,3$ such that $p+q=3$.

In the sequel we assume that $F^{\prime}(\alpha)$ is nonsingular. We can then introduce the maps

$$
A_{k}=\frac{1}{k !}\left[F^{\prime}(\alpha)\right]^{-1} F^{(k)}(\alpha) \in \mathscr{L}\left(\mathbb{R}^{m} \times \stackrel{k}{\cdot} \times \mathbb{R}^{m}, \mathbb{R}^{m}\right), \quad k=2,3,4 .
$$


Also, it will be convenient to write

$$
w_{k}(e)
$$

for any vector-valued expression in $e$ whose norm is $o\left(\|e\|^{k}\right)$; similarly we write

$$
w_{j, k}(e, \tilde{e})
$$

for any expression in $e, \tilde{e}$ such that whose norm is $o\left(\|e\|^{j}\|\tilde{e}\|^{k}\right)$. Here $j, k$ are natural numbers.

Theorem 1. The iterative procedure in (4) has $R$-order of convergence at least 2 if $\gamma+\delta=2$ and at least $\frac{1}{2}(1+\sqrt{5})$ if $\gamma+\delta \neq 2$. More precisely, if $F^{\prime}(\alpha)$ is nonsingular, then

$$
e_{n+1}=A_{2} e_{n}^{2}+(1-\gamma)^{2} A_{3} e_{n-1}^{2} e_{n}+w_{2,1}\left(e_{n-1}, e_{n}\right) \quad \text { if } \gamma+\delta=2
$$

and

$$
e_{n+1}=(2-\gamma-\delta) A_{2} e_{n-1} e_{n}+(\gamma+\delta-1) A_{2} e_{n}^{2}+w_{2}\left(e_{n-1}\right) \quad \text { if } \gamma+\delta \neq 2 .
$$

Proof. We set $y=y_{n}$ and $x=z_{n}$ in (6) to obtain the expression of $\left[y_{n}, z_{n} ; F\right]$ in terms of $e_{n}=x_{n}-\alpha$. Then, by expanding in formal power series of $e_{n-1}$ and $e_{n}$ and taking into account that $\left[y_{n}, z_{n} ; F\right]^{-1}\left[y_{n}, z_{n} ; F\right]=I$, we obtain

$$
\begin{aligned}
& {\left[y_{n}, z_{n} ; F\right]^{-1}} \\
& =\left(I-(2-\gamma-\delta) A_{2} e_{n-1}-(\gamma+\delta) A_{2} e_{n}\right. \\
& \left.\quad-\left(\left((1-\gamma)^{2}+(1-\delta)^{2}+(1-\gamma)(1-\delta)\right) A_{3}-(2-\gamma-\delta)^{2} A_{2}^{2}\right) e_{n-1}^{2}+w_{2}\left(e_{n-1}\right)\right) \\
& \quad \times\left[F^{\prime}(\alpha)\right]^{-1} .
\end{aligned}
$$

The highest local order of convergence for (4) is obtained when $\gamma+\delta=2$, since then the term $(2-\gamma-\delta) A_{2} e_{n-1}$ disappears. In this case, $\delta=2-\gamma$ and

$$
\left.\left[y_{n}, z_{n} ; F\right]^{-1}=\left(I-2 A_{2} e_{n}-(1-\gamma)^{2} A_{3} e_{n-1}^{2}\right)+w_{2}\left(e_{n-1}\right)\right)\left[F^{\prime}(\alpha)\right]^{-1},
$$

so that (4) becomes

$\left\{\begin{array}{l}x_{0}, x_{-1} \text { given in } D, \\ x_{n+1}=x_{n}-\left[\gamma x_{n}+(1-\gamma) x_{n-1},(2-\gamma) x_{n}+(\gamma-1) x_{n-1} ; F\right]^{-1} F\left(x_{n}\right), \quad n \geq 0 .\end{array}\right.$

By subtracting the root $\alpha$ from both sides of (9), we deduce that

$$
\begin{aligned}
e_{n+1}=e_{n}-\left(I-2 A_{2} e_{n}-(1-\gamma)^{2} A_{3} e_{n-1}^{2}+w_{2}\left(e_{n-1}\right)\right)[ & \left.F^{\prime}(\alpha)\right]^{-1} F^{\prime}(\alpha) \\
& \times\left(e_{n}+A_{2} e_{n}^{2}+w_{2}\left(e_{n}\right)\right),
\end{aligned}
$$

which leads to (7). Taking norms, we then have 


$$
\left\|e_{n+1}\right\| \leq\left\|A_{2}\right\|\left\|e_{n}\right\|^{2}+(1-\gamma)^{2}\left\|A_{3}\right\|\left\|e_{n-1}\right\|^{2}\left\|e_{n}\right\| .
$$

Consequently the associated equation is $t^{2}-t-2=0$ [20; 25], whose only positive root is 2 . Thus the $R$-order of convergence of family (9) is at least 2 .

In the other case, $\gamma+\delta \neq 2$, we argue as above and deduce (8) and the inequality

$$
\left\|e_{n+1}\right\| \leq|2-\gamma-\delta|\left\|A_{2}\right\|\left\|e_{n-1}\right\|\left\|e_{n}\right\|+|\gamma+\delta-1|\left\|A_{2}\right\|\left\|e_{n}\right\|^{2} .
$$

The associated equation is now $t^{2}-t-1=0$, whose unique positive root is $\frac{1}{2}(1+\sqrt{5})$. Thus the $R$-order of convergence is at least $\frac{1}{2}(1+\sqrt{5})$, which is that of the secant method.

\section{Efficiency}

We next turn to the efficiency of the two-parameter family of iterative methods (4), comparing it with that of the one-parameter family (3). Having just determed the $R$-orders of convergence, we need to find the number of function evaluations and operations (multiplications and divisions) required at each step.

We denote by $a_{1}(m)$ and $p_{1}(m)$, respectively, the number of function evaluations and operations (per step) for (3) in dimension $m$. For the two-parameter family (4), the corresponding numbers are denoted by $a_{2}(m)$ and $p_{2}(m)$ in the case $\gamma+\delta \neq 2$, and by $a_{3}(m)$ and $p_{3}(m)$ in the case $\gamma+\delta=2$.

To determine $a_{1}(m)$ and $p_{1}(m)$, we rewrite the last line of (3) as

$$
x_{n+1}=x_{n}+b_{n}, \quad \text { where }\left[y_{n}, x_{n} ; F\right] b_{n}=-F\left(x_{n}\right) .
$$

We see that $m$ evaluations are needed for the $F_{i}$ and $m^{2}$ for functions in the divided difference matrix, so

$$
a_{1}(m)=m^{2}+m \text {. }
$$

Also needed are $m^{2}+2 m$ operations to compute the divided difference matrix (counting $y_{n}=\lambda x_{n}+(1-\lambda) x_{n+1}$ as two multiplications), $\frac{1}{3}\left(m^{3}-m\right)$ operations for its LU decomposition, and $m^{2}$ operations to solve two triangular linear systems. Therefore

$$
p_{1}(m)=\frac{1}{3} m\left(m^{2}+6 m+5\right) .
$$

With these two values we can then compute the two measures of efficiency,

$$
\mathrm{EI}=\left(\frac{1+\sqrt{5}}{2}\right)^{1 / a_{1}(m)} \text { and } \mathrm{CE}=\left(\frac{1+\sqrt{5}}{2}\right)^{1 / p_{1}(m)} .
$$

Similarly, for (4) with $\gamma+\delta \neq 2$, we can write

$$
x_{n+1}=x_{n}+c_{n}, \quad \text { where }\left[y_{n}, z_{n} ; F\right] c_{n}=-F\left(x_{n}\right) .
$$



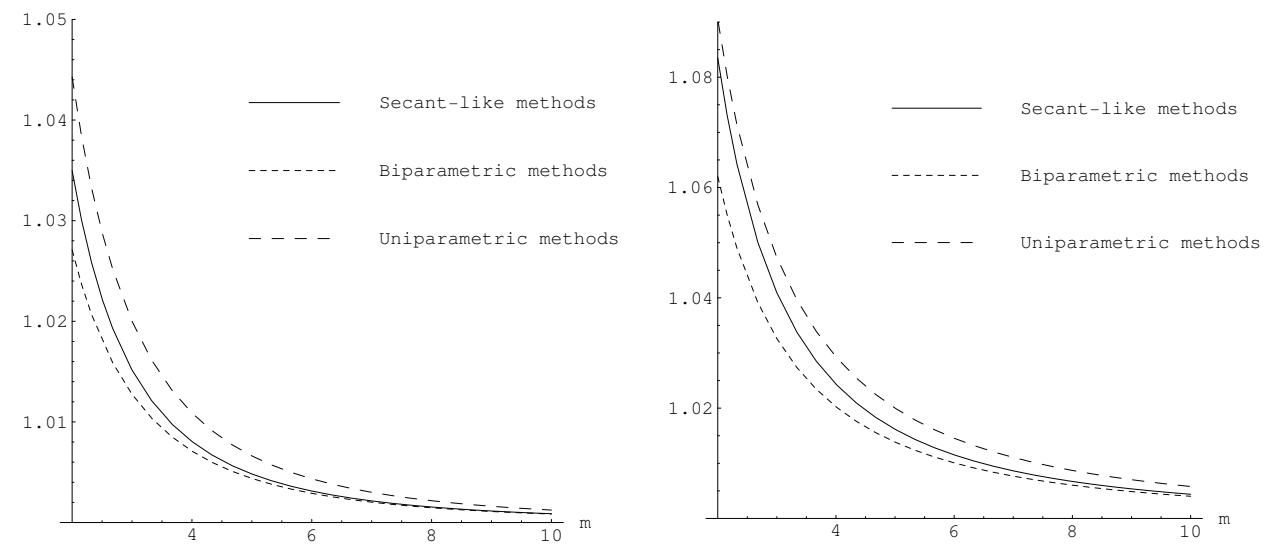

Figure 1. Plots of EI (left) and CE (right) versus the dimension $m$. The bottom curves refer to the general two-parameter algorithm (4); the efficiency indices are given in (11). The middle curves refer to the "secant-like" specialization $(\delta=0)$; see (13). The top curves refer to the specialization $\gamma+\delta=2$; see (14).

In this case we get $a_{2}(m)=a_{1}(m)+m=m^{2}+2 m$ function evaluations and $p_{2}(m)=p_{1}(m)+2 m=\frac{1}{3} m\left(m^{2}+6 m+8\right)$ operations (because $z_{n}$, too, requires two multiplications). This leads to

$$
\mathrm{EI}=\left(\frac{1+\sqrt{5}}{2}\right)^{1 /\left(a_{1}(m)+m\right)} \text { and } \mathrm{CE}=\left(\frac{1+\sqrt{5}}{2}\right)^{1 /\left(p_{1}(m)+2 m\right)} .
$$

Finally we take (4) with $\gamma+\delta=2$. Equation (12) is still valid; however, since $\left.z_{n}=(2-\gamma) x_{n}+(\gamma-1) x_{n-1}\right)$ shares a summand with $y_{n}$, it requires one fewer multiplication. Consequently, $a_{3}(m)=a_{1}(m)+m=m^{2}+2 m$ and $p_{3}(m)=$ $p_{1}(m)+m=\frac{1}{3} m\left(m^{2}+6 m+8\right)$. In this case we obtain

$$
\mathrm{EI}=2^{1 /\left(a_{1}(m)+m\right)} \quad \text { and } \quad \mathrm{CE}=2^{1 /\left(p_{1}(m)+m\right)} .
$$

The results are summarized in Figure 1. We see that both measures of efficiency, the EI the the CE, are highest for the case $\gamma+\delta=2$ of the two-parameter family (4). Within this spacial case, the Kurchatov method $(\gamma=0, \delta=2)$ is the most efficient of all, since it saves $m$ function evaluations and $3 m$ multiplications.

\section{Applications}

As already discussed, the EI and CE are based, respectively, on the number of function evaluations and the number of operations. To take both into account at once we can use what we call the computational efficiency index, defined as

$$
\mathrm{CEI}=\rho^{1 / \mathscr{C}} .
$$


Here $\rho$ is the $R$-order of convergence and $\mathscr{C}$ is the computational cost per step, defined as the number of operations plus $\mu$ times the number of function evaluations. The factor $\mu$ reflects the cost of a function evaluation relative to that of an operation, and depends on the machine, the software and the arithmetic used. (Some discussion of the CEI can be found in [22].) In Section 4.1 we use the CEI to refine the analysis of the previous section. In Section 4.2 we illustrate with Troesch's problem [26].

4.1. Optimal computational efficiency. We have seen in Section 3 that two special cases stand out for efficiency among the algorithm of the family (4): the secant method $(2)$, with $(\gamma, \delta)=(0,1)$, and the Kurchatov method $(5)$, with $(\gamma, \delta)=(0,2)$. Combining the definition of $\mathscr{C}$ in the previous paragraph with the results of Section 3 , we have

$$
\begin{aligned}
\mathscr{C}^{\mathrm{sec}}(\mu, m) & =m^{2} \mu+\frac{1}{3} m\left(m^{2}+6 m-1\right), & & \rho^{\mathrm{sec}}=\frac{1}{2}(1+\sqrt{5}), \\
\mathscr{C}^{\mathrm{Kur}}(\mu, m) & =\left(m^{2}+m\right) \mu+\frac{1}{3} m\left(m^{2}+6 m-1\right), & & \rho^{\mathrm{Kur}}=2 .
\end{aligned}
$$

Theorem 2. If $m=2$, then $\mathrm{CEI}_{\mathrm{sec}}>\mathrm{CEI}_{\mathrm{Kur}}$ for $\mu>\mu_{0}: \approx 18.48023$, and $\mathrm{CEI}_{\mathrm{Kur}}>\mathrm{CEI}_{\text {sec }}$ for $\mu<\mu_{0}$. If $m \geq 3$, then $\mathrm{CEI}_{\mathrm{Kur}}>\mathrm{CEI}_{\text {sec }}$.

Proof. It is enough to consider the borderline case of the ratio

$$
\frac{\log \mathrm{CEI}^{\mathrm{sec}}}{\log \mathrm{CEI}^{\mathrm{Kur}}}=\frac{\mathscr{C}^{\mathrm{Kur}}}{\mathscr{C}^{\mathrm{sec}}} \frac{\log \rho^{\mathrm{sec}}}{\log \rho^{\mathrm{Kur}}} .
$$

Equating this ratio to 1 gives a curve in the $(m, \mu)$ plane with vertical asymptote $m=2.270559 \ldots$ For higher $m$, the ratio is always less than 1 . For $m=2$, the ratio is less than 1 if and only if $\mu>\mu_{0}$.

Therefore, the CEI of the Kurchatov method is almost always better than that of the secant method.

4.2. Troesch's problem. Troesch's problem [26] is the following nonlinear twopoint boundary value problem in one dimension:

$$
u^{\prime \prime}(x)=\lambda \sinh (\lambda u(x)), \quad 0 \leq x \leq 1,
$$

with boundary conditions $u(0)=0$ and $u(1)=1$ and the real positive parameter $\lambda$. It arises from modeling the confinement of a plasma column by radiation pressure. A closed-form solution to the problem is known $[6 ; 7 ; 9 ; 17 ; 23]$. It can be written in terms of the Jacobian elliptic function sc as

$$
u(x)=\frac{2}{\lambda} \sinh ^{-1}\left\{12 u^{\prime}(0) \operatorname{sc}\left(\lambda x, 1-\frac{1}{4} u^{\prime}(0)^{2}\right)\right\},
$$

where $u^{\prime}(0)$ is the derivative at $t=0$; we have $u^{\prime}(0)=2 \sqrt{1-\kappa}$, where $\kappa$ is the 


\begin{tabular}{|ccccc|}
\hline digits & $x * y$ & $x / y$ & $\sqrt{x}$ & $\exp (x)$ \\
\hline 32 & $1.1 \mu \mathrm{s}$ & 1 & 11 & 25 \\
\hline
\end{tabular}

Table 1. Estimated computational cost of elementary functions computed with Maple@ 13 on an Intel ${ }^{\circledR}$ Core $^{\mathrm{TM}} 2$ Duo CPU P8800 (32-bit machine) running Microsoft Windows 7 Professional, where $x=\sqrt{3}-1$ and $y=\sqrt{5}$. The last three entries are relative to the second (multliplication).

solution to the equation

$$
\frac{\sinh (\lambda / 2)}{\sqrt{1-\kappa}}=\operatorname{sc}(\lambda, \kappa) .
$$

Given a value of $\lambda$, we can find $\kappa$ from (18) and the defining equation $\operatorname{sc}(\lambda, \kappa)=$ $\tan \phi$, where $\phi$ is determined by

$$
\int_{0}^{\phi} \frac{d \theta}{\sqrt{1-\kappa \sin ^{2} \theta}}=\lambda
$$

(see [1]). Following [7; 9] we consider two cases: $\lambda=0.5$ and $\lambda=1$.

In the remainder of this section we use two finite-difference schemes to solve Troesch's problem numerically, using the closed-form solution for comparison. The numerical computations were performed using Maple with 32 digits. To specify the computational cost, an estimation of the factor $\mu$ is necessary. We used the data in Table 1, based on $[10 ; 19]$.

A classic finite difference scheme. We partition the interval $[0,1]$ as follows:

$$
x_{0}=0<x_{1}<x_{2}<\cdots<x_{n-1}<x_{n}=1, \quad x_{j+1}=x_{j}+h, \quad h=1 / n,
$$

and define $y_{0}=y\left(x_{0}\right)=0, y_{1}=y\left(x_{1}\right), \ldots, y_{n-1}=y\left(x_{n-1}\right), y_{n}=y\left(x_{n}\right)=1$. If we discretize (16) by using the standard numerical formula for the second derivative,

$$
y_{k}^{\prime \prime}=\frac{y_{k-1}-2 y_{k}+y_{k+1}}{h^{2}}+O\left(h^{2}\right), \quad k=1,2, \ldots, n-1,
$$

we obtain the following system of $(n-1) \times(n-1)$ nonlinear equations:

$$
y_{k-1}-\left(2 y_{k}+h^{2} \lambda \sinh \left(\lambda y_{k}\right)\right)+y_{k+1}=0, \quad k=1,2, \ldots, n-1 .
$$

Setting $n=20$, the approximate solution is computed taking the initial points $\boldsymbol{x}_{-1}=(1,1, \ldots, 1)$ and $\boldsymbol{x}_{0}=(0,0, \ldots, 0)$ and applying methods (2) and (5), the secant and Kurchatov methods.

The errors in the solution are shown in Table 2 and more information about efficiencies is shown in Table 3. The cost is computed in the following way: the cost of the function sinh is 27 ( 25 for the exponential function plus 2 divisions); each component function $F_{k}$ is equal to $y_{k-1}-\left(2 y_{k}+h^{2} \lambda \sinh \left(\lambda y_{k}\right)\right)+y_{k+1}$ with 


\begin{tabular}{|c|ccc|ccc|}
\hline & \multicolumn{3}{|c|}{$\lambda=0.5$} & \multicolumn{3}{|c|}{$\lambda=1.0$} \\
\hline$x$ & $u(x)$ & $|u(x)-y(x)|$ & $|u(x)-z(x)|$ & $u(x)$ & $|u(x)-y(x)|$ & $|u(x)-z(x)|$ \\
\hline 0.1 & 0.095944349292 & $4.1627 \cdot 10^{-7}$ & $3.4372 \cdot 10^{-12}$ & 0.084661256551 & $5.9888 \cdot 10^{-6}$ & $5.6178 \cdot 10^{-11}$ \\
0.2 & 0.192128747660 & $8.0952 \cdot 10^{-7}$ & $6.6447 \cdot 10^{-12}$ & 0.170171358178 & $1.1732 \cdot 10^{-5}$ & $1.0262 \cdot 10^{-10}$ \\
0.3 & 0.288794400893 & $1.1563 \cdot 10^{-6}$ & $9.3965 \cdot 10^{-12}$ & 0.257393908080 & $1.6965 \cdot 10^{-5}$ & $1.3041 \cdot 10^{-10}$ \\
0.4 & 0.386184846362 & $1.4323 \cdot 10^{-6}$ & $1.1475 \cdot 10^{-11}$ & 0.347222855110 & $2.1385 \cdot 10^{-5}$ & $1.3243 \cdot 10^{-10}$ \\
0.5 & 0.484547164744 & $1.6118 \cdot 10^{-6}$ & $1.2675 \cdot 10^{-11}$ & 0.440599835168 & $2.4626 \cdot 10^{-5}$ & $1.0472 \cdot 10^{-10}$ \\
0.6 & 0.584133248445 & $1.6674 \cdot 10^{-6}$ & $1.2810 \cdot 10^{-11}$ & 0.538534398077 & $2.6221 \cdot 10^{-5}$ & $4.8544 \cdot 10^{-11}$ \\
0.7 & 0.685201148302 & $1.5690 \cdot 10^{-6}$ & $1.1717 \cdot 10^{-11}$ & 0.642128609191 & $2.5561 \cdot 10^{-5}$ & $2.6357 \cdot 10^{-11}$ \\
0.8 & 0.788016522650 & $1.2837 \cdot 10^{-6}$ & $9.2672 \cdot 10^{-12}$ & 0.752608094046 & $2.1818 \cdot 10^{-5}$ & $9.6507 \cdot 10^{-11}$ \\
0.9 & 0.892854216136 & $7.7458 \cdot 10^{-7}$ & $5.3721 \cdot 10^{-12}$ & 0.871362519798 & $1.3843 \cdot 10^{-5}$ & $1.1578 \cdot 10^{-10}$ \\
\hline
\end{tabular}

Table 2. Exact and approximate solutions $u(x), y(x)$ and $z(x)$ defined in (17), (21) and (22), respectively.

\begin{tabular}{|c|c|c|c|c|c|c|c|c|c|c|}
\hline$\lambda=0.5$ & & & & & & & & & & \\
\hline & $I$ & $a$ & $a \mu$ & $v$ & $\mathscr{b}$ & EI & $\mathrm{CE}$ & CEI & $\mathrm{TF}$ & $\tau$ \\
\hline method (2) & 3 & $3 m$ & $87 m$ & $6 m-4$ & 1763 & 1.0084780 & 1.0043842 & 1.0002730 & 8435.91 & 0.024516 \\
\hline method (5) & 2 & $3 m$ & $87 m$ & $6 m-4$ & 1763 & 1.0122347 & 1.0063212 & 1.0003932 & 5856.56 & 0.018875 \\
\hline \multicolumn{11}{|l|}{$\lambda=1.0$} \\
\hline & $I$ & $a$ & $a \mu$ & $v$ & $\mathscr{C}$ & EI & $\mathrm{CE}$ & CEI & $\mathrm{TF}$ & $\tau$ \\
\hline method (2) & 3 & $3 m$ & $84 m$ & $6 m-4$ & 1706 & 1.0084780 & 1.0043842 & 1.0002821 & 8163.16 & 0.024438 \\
\hline method (5) & 2 & $3 m$ & $84 m$ & $6 m-4$ & 1706 & 1.0122347 & 1.0063212 & 1.0004064 & 5667.21 & 0.019000 \\
\hline
\end{tabular}

Table 3. Numerical efficiency for system (21) with $m=19$.

$h^{2}=1 / 400$ and $h^{2} \lambda$ prefixed, so that we have an evaluation of $\sinh$ and 2 products if $\lambda=0.5$ (in total 29), whereas we have an evaluation of sinh and 1 product (in total 28) if $\lambda=1$. In short, $\mu_{\lambda=0.5}=29$ and $\mu_{\lambda=1}=28$.

A nonstandard finite difference scheme. As a consequence of the low accuracy obtained in the previous section, we now discretize (16) in a different way. We again consider the partition of the interval $[0,1]$ given in (19), define $z_{0}=z\left(x_{0}\right)=0$, $z_{1}=z\left(x_{1}\right), \ldots, z_{n-1}=z\left(x_{n-1}\right), z_{n}=z\left(x_{n}\right)=1$ and discretize (16) by using the following smart numerical formula for the second derivative [7]:

$$
z_{k}^{\prime \prime}=\frac{w_{k}^{2}\left(z_{k-1}-2 z_{k}+z_{k+1}\right)}{2\left(\cosh \left(w_{k} h\right)-1\right)}+O\left(h^{4}\right), \quad k=1,2, \ldots, n-1,
$$

where

$$
w_{k}=\lambda \sqrt{\frac{\left(z_{k+1}-z_{k-1}\right)^{2}}{4 h^{2}}+\cosh \left(\lambda z_{k}\right)}
$$


Next, we obtain the following system of $(n-1) \times(n-1)$ nonlinear equations:

$$
w_{k}^{2}\left(z_{k+1}-2 z_{k}+z_{k-1}\right)-2 \lambda \sinh \left(\lambda z_{k}\right)\left(\cosh \left(w_{k} h\right)-1\right)=0, \quad k=1, \ldots, n-1 .
$$

Setting $n=20$, the approximate solution is computed taking the initial points

$\boldsymbol{x}_{-1}=(.0480, .0959, .144, .192, .240, .289, .337, .386, .435, .485$,

$.534, .584, .634, .685, .736, .788, .840, .893, .946)^{t}$,

and

$\boldsymbol{x}_{0}=(.047957, .095944, .14399, .19213, .24039, .28879, .33738, .38618, .43523, .48455$, $.53417, .58413, .63447, .68520, .73637, .78802, .84016, .89285, .94612)^{t}$,

and applying again methods (2) and (5). The errors in the solution are shown in Table 2 and more information about efficiencies is given in Table 4 . The cost of the function cosh is the same as that of sinh if this function is not computed before. In this case, the cost is 1 . Every component function $F_{k}$ is equal to $w_{k}^{2}\left(z_{k+1}-2 z_{k}+z_{k-1}\right)-2 \lambda \sinh \left(\lambda z_{k}\right)\left(\cosh \left(h w_{k}\right)-1\right), w_{k}^{2}$ with cost equal to 31 or $29, w_{k}$ with cost equal to $11, \sinh \left(\lambda z_{k}\right)$ with cost equal to 28 or $27, \cosh \left(w_{k} h\right)$ with cost equal to $39(27$ (cosh) +11 (sqrt) +1 (prod)), and some isolated products. Finally, we obtain $\mu_{\lambda=0.5}=73$ and $\mu_{\lambda=1}=72$.

In Table 2 , for $\lambda=0.5$ and $\lambda=1$, we present the exact solution $u\left(x_{\ell}\right)$, the numerical solution $y\left(x_{\ell}\right)$ of (21) and the numerical solution $z\left(x_{\ell}\right)$ of (22), where $\ell=1,2, \ldots, 9$ and $k=2 \ell$, which $k$ is given in (21) and (22). In both cases the results are independent of the application of methods (2) and (5).

Table 2 confirms the theoretical results. It is interesting that inaccurate tabulated "exact" solutions are given in $[9 ; 18]$, but those numerical results would approximate exact results more closely if their calculations of the later where properly done.

Tables 3 and 4 show the results obtained for both methods. In each table we show the number of iterations, $I$, needed to get the required precision, the computational $\operatorname{cost} \mathscr{C}$, the computational efficiency index CEI defined in (15) and the time factor $T F$ defined by $1 / \log (\mathrm{CEI})$. If the values of the CEI are so close as to be almost indistinguishable in practice, we can then observe the $T F$ that tell better the difference between iterative methods. While in the definition of the CEI we have considered functions with the divided difference full of terms, we observe that the two given discretizations to solve Troesch's problem provide a tridiagonal operator.

If both methods are applied to solve systems of $m$ nonlinear equations, we have to solve a triangular linear system per iteration, $2(m-1)$ operations (products and divisions) are required in the LU decomposition, $2 m-1$ operations in the backward substitution and $m-1$ operations in the forward substitution. Therefore, the number 


\begin{tabular}{|c|c|c|c|c|c|c|c|c|c|c|}
\hline \multicolumn{11}{|l|}{$\lambda=0.5$} \\
\hline & $I$ & $a$ & $a \mu$ & $v$ & $\mathscr{b}$ & EI & $\mathrm{CE}$ & CEI & TF & $\tau$ \\
\hline method (2) & 5 & $5 m-2$ & $73(5 m-2)$ & $8 m-6$ & 7028 & 1.005188 & 1.003301 & 1.000069 & 33183.78 & .155234 \\
\hline method (5) & 5 & $5 m-2$ & $73(5 m-2)$ & $8 m-6$ & 7028 & 1.007481 & 1.004759 & 1.000099 & 23163.80 & .147970 \\
\hline \multicolumn{11}{|l|}{$\lambda=1.0$} \\
\hline & $I$ & $a$ & $a \mu$ & $v$ & $\mathscr{b}$ & EI & $\mathrm{CE}$ & CEI & TF & $\tau$ \\
\hline method (2) & 5 & $5 m-2$ & $72(5 m-2)$ & $8 m-6$ & 6842 & 1.005188 & 1.003301 & 1.000070 & 32738.78 & .151870 \\
\hline method (5) & 4 & $5 m-2$ & $72(5 m-2)$ & $8 m-6$ & 6842 & 1.007481 & 1.004759 & 1.000101 & 22728.63 & .131109 \\
\hline
\end{tabular}

Table 4. Numerical efficiency for system (22) with $m=19$.

of operations needed per iteration is $5 m-4$ for both methods. The number of function evaluations is computed in the following way:

- We have $m$ evaluations of the function $F: F_{1}, F_{2}, \ldots, F_{m}$.

- For the classic finite difference scheme, we have $2 m$ evaluations and $m$ divisions to evaluate the divided difference, so that $\mathscr{b}=3 m \mu+6 m-4$.

- For the nonstandard finite difference scheme, we have to compute $4 m-2$ evaluations and $3 m-2$ divisions in the divided difference matrix, so that $\mathscr{C}=(5 m-2) \mu+8 m-6$.

Tables 3 and 4 confirm the theoretical results. For Troesch's problem, the costs are the same and we then observe that method (5) has the highest value of CEI in all cases, since $\mathrm{CEI}^{\mathrm{Kur}}>\mathrm{CEI}^{\mathrm{sec}}$, which confirms the results of Section 4.1.

\section{Concluding remarks}

We present a two-parameter family of iterative methods for solving nonlinear systems of equations with local $R$-order of convergence higher than other competitive iterative methods. Between the members of the family we point out the Kurchatov method and the secant method. Moreover, we analyze a generalization of the efficiency used in the one-dimensional case to several variables. Finally, we show an application, where Troesch's problem is considered, which illustrates the theoretical results presented in the paper and conclude that the Kurchatov method is more efficient than the secant method for solving Troesch's problem.

\section{References}

[1] M. Abramowitz and I. A. Stegun, Handbook of mathematical functions with formulas, graphs, and mathematical tables, National Bureau of Standards Applied Mathematics Series, no. 55, U.S. Government Printing Office, Washington, DC, 1964, Reprinted by Dover, New York, 1974. MR 29 \#4914 Zbl 0171.38503 
[2] I. K. Argyros, The secant method and fixed points of nonlinear operators, Monatsh. Math. 106 (1988), no. 2, 85-94. MR 90b:65111 Zbl 0652.65043

[3] - On the secant method, Publ. Math. Debrecen 43 (1993), no. 3-4, 223-238. MR 95j: 47077 Zbl 0796.65075

[4] On a two-point Newton-like method of convergent order two, Int. J. Comput. Math. 82 (2005), no. 2, 219-233. MR 2158994 Zbl 1068.65070

[5] _ A Kantorovich-type analysis for a fast iterative method for solving nonlinear equations, J. Math. Anal. Appl. 332 (2007), no. 1, 97-108. MR 2008g:65075 Zbl 1121.65061

[6] J. P. Boyd, One-point pseudospectral collocation for the one-dimensional Bratu equation, Appl. Math. Comput. 217 (2011), no. 12, 5553-5565. MR 2770174 Zbl 1222.65070

[7] U. Erdogan and T. Ozis, A smart nonstandard finite difference scheme for second order nonlinear boundary value problems, J. Comput. Phys. 230 (2011), no. 17, 6464-6474. MR 2012m:65218 Zbl 05992164

[8] J. A. Ezquerro, M. Grau-Sánchez, A. Grau, M. A. Hernández, M. Noguera, and N. Romero, On iterative methods with accelerated convergence for solving systems of nonlinear equations, J. Optim. Theory Appl. 151 (2011), no. 1, 163-174. MR 2012j:65145 Zbl 1226.90103

[9] X. Feng, L. Mei, and G. He, An efficient algorithm for solving Troesch's problem, Appl. Math. Comput. 189 (2007), no. 1, 500-507. MR 2330227 Zbl 1122.65373

[10] L. Fousse, G. Hanrot, V. Lefèvre, P. Pélissier, and P. Zimmermann, MPFR: a multiple-precision binary floating-point library with correct rounding, ACM Trans. Math. Software 33 (2007), no. 2, Article ID \#13. MR 2008e:65157

[11] M. Grau-Sánchez, À. Grau, and M. Noguera, Frozen divided difference scheme for solving systems of nonlinear equations, J. Comput. Appl. Math. 235 (2011), no. 6, 1739-1743. MR 2012a:65132 Zbl 1204.65051

[12] M. Grau-Sánchez and M. Noguera, A technique to choose the most efficient method between secant method and some variants, Appl. Math. Comput. 218 (2012), no. 11, 6415-6426. MR 2879122 Zbl 06036020

[13] M. A. Hernández and M. J. Rubio, A uniparametric family of iterative processes for solving nondifferentiable equations, J. Math. Anal. Appl. 275 (2002), no. 2, 821-834. MR 2003i:47077 Zbl 1019.65036

[14] M. A. Hernández, M. J. Rubio, and J. A. Ezquerro, Secant-like methods for solving nonlinear integral equations of the Hammerstein type, J. Comput. Appl. Math. 115 (2000), no. 1-2, 245-254. MR 2000m:65157 Zbl 0944.65146

[15] _ Solving a special case of conservative problems by secant-like methods, Appl. Math. Comput. 169 (2005), no. 2, 926-942. MR 2006g:65078 Zbl 1080.65044

[16] V. A. Kurčatov, A certain linear interpolation method for solving functional equations, Dokl. Akad. Nauk SSSR 198 (1971), 524-526, In Russian; translated in Soviet Math. Dokl. 12 (1971) 835-838. MR 45 \#6211 Zbl 0252.65044

[17] Y. Lin, J. A. Enszer, and M. A. Stadtherr, Enclosing all solutions of two point boundary value problems for ODE's, Comput. Chem. Eng. 32 (2008), 1714-1725.

[18] S. T. Mohyud-Din, Solution of Troesch's problem using He's polynomials, Rev. Un. Mat. Argentina 52 (2011), no. 1, 143-148. MR 2815720 Zbl 05965157

[19] The MPFR library 2.2.0: timings.

[20] A. M. Ostrowski, Solutions of equations and systems of equations, Pure and Applied Mathematics, no. 9, Academic Press, New York, 1960. MR 23 \#B571 Zbl 0115.11201 
[21] F. A. Potra, On Q-order and R-order of convergence, J. Optim. Theory Appl. 63 (1989), no. 3, 415-431. MR 91d:65077 Zbl 0663.65049

[22] F. A. Potra and V. Pták, Nondiscrete induction and iterative processes, Research Notes in Mathematics, no. 103, Pitman, Boston, MA, 1984. MR 86i:65003 Zbl 0549.41001

[23] S. M. Roberts and J. S. Shipman, On the closed form solution of Troesch's problem, J. Comput. Phys. 21 (1976), no. 3, 291-304. MR 54 \#4122 Zbl 0334.65062

[24] S. M. Shakhno, On a Kurchatov's method of linear interpolation for solving nonlinear equations, Proc. Appl. Math. Mech. 4 (2004), 650-651.

[25] J. F. Traub, Iterative methods for the solution of equations, Prentice-Hall, Englewood Cliffs, NJ, 1964. MR 29 \#6607 Zbl 0121.11204

[26] B. A. Troesch, A simple approach to a sensitive two-point boundary value problem, J. Comput. Phys. 21 (1976), no. 3, 279-290. MR 54 \#4121 Zbl 0334.65063

Received March 29, 2012. Revised October 15, 2012.

José A. EZQUERRo: jezquer@unirioja.es

Department of Mathematics and Computation, University of La Rioja, 26004 Logroño, Spain

ANGELA GRAU: angela.grau@upc.edu

Department of Applied Mathematics II, Technical University of Catalonia, 08034 Barcelona, Spain

MiQuel GraU-SÁNCHEZ: miquel.grau@upc.edu

Department of Applied Mathematics II, Technical University of Catalonia, 08034 Barcelona, Spain

Miguel A. HernándeZ-VERón: mahernan@unirioja.es

Department of Mathematics and Computation, University of La Rioja, 26004 Logroño, Spain 


\title{
Communications in Applied Mathematics and Computational Science
}

\author{
msp.org/camcos
}

EDITORS

MANAGING EDITOR

John B. Bell

Lawrence Berkeley National Laboratory, USA

jbbell@lbl.gov

\section{BOARD OF EDITORS}

\begin{tabular}{|c|c|c|c|}
\hline Marsha Berger & $\begin{array}{l}\text { New York University } \\
\text { berger@cs.nyu.edu }\end{array}$ & Ahmed Ghoniem & $\begin{array}{l}\text { Massachusetts Inst. of Technology, USA } \\
\text { ghoniem@mit.edu }\end{array}$ \\
\hline Alexandre Chorin & $\begin{array}{l}\text { University of California, Berkeley, USA } \\
\text { chorin@math.berkeley.edu }\end{array}$ & Raz Kupferman & $\begin{array}{l}\text { The Hebrew University, Israel } \\
\text { raz@math.huji.ac.il }\end{array}$ \\
\hline Phil Colella & $\begin{array}{l}\text { Lawrence Berkeley Nat. Lab., USA } \\
\text { pcolella@lbl.gov }\end{array}$ & Randall J. LeVeque & $\begin{array}{l}\text { University of Washington, USA } \\
\text { rj1@ amath.washington.edu }\end{array}$ \\
\hline Peter Constantin & $\begin{array}{l}\text { University of Chicago, USA } \\
\text { const@cs.uchicago.edu }\end{array}$ & Mitchell Luskin & $\begin{array}{l}\text { University of Minnesota, USA } \\
\text { luskin@umn.edu }\end{array}$ \\
\hline Maksymilian Dryja & $\begin{array}{l}\text { Warsaw University, Poland } \\
\text { maksymilian.dryja@acn.waw.pl }\end{array}$ & Yvon Maday & $\begin{array}{l}\text { Université Pierre et Marie Curie, France } \\
\text { maday@ann.jussieu.fr }\end{array}$ \\
\hline M. Gregory Forest & $\begin{array}{l}\text { University of North Carolina, USA } \\
\text { forest@amath.unc.edu }\end{array}$ & James Sethian & $\begin{array}{l}\text { University of California, Berkeley, USA } \\
\text { sethian@ math.berkeley.edu }\end{array}$ \\
\hline Leslie Greengard & $\begin{array}{l}\text { New York University, USA } \\
\text { greengard@ cims.nyu.edu }\end{array}$ & Juan Luis Vázquez & $\begin{array}{l}\text { Universidad Autónoma de Madrid, Spain } \\
\text { juanluis.vazquez@uam.es }\end{array}$ \\
\hline Rupert Klein & $\begin{array}{l}\text { Freie Universität Berlin, Germany } \\
\text { rupert.klein@pik-potsdam.de }\end{array}$ & Alfio Quarteroni & $\begin{array}{l}\text { Ecole Polytech. Féd. Lausanne, Switzerland } \\
\text { alfio.quarteroni@epfl.ch }\end{array}$ \\
\hline \multirow[t]{2}{*}{ Nigel Goldenfeld } & $\begin{array}{l}\text { University of Illinois, USA } \\
\text { nigel@uiuc.edu }\end{array}$ & Eitan Tadmor & $\begin{array}{l}\text { University of Maryland, USA } \\
\text { etadmor@cscamm.umd.edu }\end{array}$ \\
\hline & & Denis Talay & $\begin{array}{l}\text { INRIA, France } \\
\text { denis.talay@inria.fr }\end{array}$ \\
\hline
\end{tabular}

\section{PRODUCTION}

production@msp.org

Silvio Levy, Scientific Editor

See inside back cover or msp.org/camcos for submission instructions.

The subscription price for 2014 is US \$75/year for the electronic version, and \$105/year ( $\$ 15$, if shipping outside the US) for print and electronic. Subscriptions, requests for back issues from the last three years and changes of subscribers address should be sent to MSP.

Communications in Applied Mathematics and Computational Science (ISSN 2157-5452 electronic, 1559-3940 printed) at Mathematical Sciences Publishers, 798 Evans Hall \#3840, c/o University of California, Berkeley, CA 94720-3840, is published continuously online. Periodical rate postage paid at Berkeley, CA 94704, and additional mailing offices.

CAMCoS peer review and production are managed by EditFLOW ${ }^{\circledR}$ from MSP.

\section{PUBLISHED BY}

mathematical sciences publishers

nonprofit scientific publishing

http://msp.org/

(C) 2014 Mathematical Sciences Publishers 


\section{Communications in Applied Mathematics and Computational Science}

vol. 9

no. 2

2014

A comparison of high-order explicit Runge-Kutta, extrapolation, and deferred correction methods in serial and parallel

DAVID I. KeTCHESON and UMAIR BIN WAHEED

A new class of secant-like methods for solving nonlinear systems of equations 201

José A. Ezquerro, Angela Grau, Miquel Grau-Sánchez and Miguel A. Hernández-Verón 